\title{
Management and Treatment of Myocarditis in Athletes
}

\section{Benjamin Hurwitz, MD Omar Issa, $D O^{*}$}

\author{
Address \\ *Department of Cardiology, Mount Sinai-Miami, Miami Beach, FL, USA \\ Email: Drissa@cgcardiology.com \\ Published online: 4 November 2020 \\ (C) Springer Science+Business Media, LLC, part of Springer Nature 2020
}

This article is part of the Topical Collection on Sports Cardiology

Keywords Myocarditis · Cardiomyopathy · Athletes · Sports eligibility · Return to play · COVID 19

Abbreviations SCD Sudden cardiac death - GCM Giant-cell myocarditis - EMB Endomyocardial biopsy - CMR Cardiac magnetic resonance - T2w T2-weighted - LGE Late gadolinium enhancement - COVID-19 SARS-Coronavirus-2

\begin{abstract}
Purpose of review Myocarditis is an inflammation of the myocardium that can often be associated with cardiac dysfunction and arrhythmias, and is even one of the leading causes for sudden cardiac death (SCD) in athletes. This review aims to summarize the current evidence and treatment guidelines for the management of myocarditis in the active population. Recent findings Physical exertion is likely a trigger for dangerous arrythmias and further propagates myocardial damage in athletes with myocarditis. For this reason, abstinence from sports is a critical facet of management in the initial inflammatory period. The use of cardiac magnetic resonance imaging, specifically late gadolinium enhancement, to guide return to play decisions is becoming more common in clinical practice.

Summary Establishing a stepwise approach for proper diagnosis and risk stratification, with an emphasis on contemporary cardiac magnetic resonance (CMR) imaging techniques, in myocarditis is critical. After a diagnosis of myocarditis is made, it is imperative for any athlete or highly active individual to refrain from physical exercise. Additionally, therapy for heart failure should be applied in cases of myocarditis with cardiac dysfunction. Undoubtedly, COVID 19, and its potential to cause myocarditis, is sure to change the landscape of management of this disease.
\end{abstract}

\section{Introduction}

Myocarditis is an inflammation of the myocardium that can often be associated with cardiac dysfunction and arrhythmias, and is even one of the leading causes for sudden cardiac death (SCD) in athletes [1]. The 
treatment of myocarditis is tailored to the individual patient and severity of myocardial insult. Physical exertion is likely a trigger for dangerous arrythmias when myocardial inflammation is present and for this reason is a critical aspect of management, with the goal of preventing adverse cardiac events $[2,3 \bullet]$. While necessary, these restrictions can be difficult to implement in a competitive athletic population.

\section{Etiology}

Myocarditis has a variety of etiologies including viruses, bacteria, drug reactions, radiation, and even autoimmune diseases. The growing use of molecular techniques, such as polymerase chain reaction amplification, suggests that viral causes of myocarditis, including enterovirus, adenovirus, influenza viruses, human herpes virus-6, cytomegalovirus, parvovirus B19, and Epstein-Barr virus, are the most common [1]. However, current evidence indicates that obtaining viral serologies is of minimal clinical significance overall. In a prospective study by Mahfoud and colleagues, only 5 of 124 patients had a viral serology consistent with the virus that was detected on endomyocardial biopsy (EMB) [4]. In this investigation, the sensitivity and specificity of viral serology were found to be $9 \%$ and $77 \%$ respectively [4]. Myocarditis from bacterial, fungal, and protozoal infections occurs with much less frequency [5]. In athletes, it is important to consider that the type of exercise being performed may predispose patients to certain pathogens. For instance, it is known that cross-country runners in certain parts of the USA are more likely to be exposed to tick bite

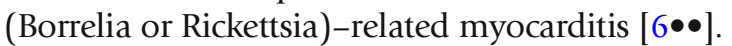

Noninfectious causes of myocarditis are less common. Inflammatory myocardial disease can also develop in the setting of giant-cell myocarditis (GCM) and sarcoidosis, both of which are rare diseases with worse overall outcomes [5]. Additionally, many medications can induce myocardial inflammation in the form of a hypersensitivity myocarditis. These medications include penicillins, antipsychotics such as clozapine, and sulfonamides [5]. Also it is imperative to complete a comprehensive patient history including assessment for the use of illicit drugs such as cocaine and anabolic steroids, which have both been shown to illicit myocardial inflammation.

It is important to note that, due the invasive nature of $\mathrm{EMB}$, the etiology of suspected myocarditis is infrequently confirmed in clinical practice [1]. In the USA, EMB is only recommended in patients with rapidly progressive or unexplained cardiomyopathy, where active myocarditis, specifically GCM, is suspected [7].

\section{Pathophysiology and the impact of exercise}

Myocarditis typically begins with an early phase of viral replication within myocytes, followed by a subacute immunologic response including both $\mathrm{T}$ cells and B cells [8]. It is here that the host's immune system, through cytokine activation and antibodies to viral proteins, can aggravate myocardial damage [5]. In the chronic phase of myocarditis, the development of fibrosis and dilatation of the cardiac chambers ensue [5]. This may manifest as cardiac 


\section{Clinical manifestations}

dysfunction and fulminant heart failure. However, more often than not, patients experience complete myocardial recovery.

The impact of exercise on immune function may have an important role in the athlete's biologic response to myocarditis. Moderate intensity exercise can vastly improve a person's immune response such that those who regularly exercise can benefit from heightened vaccine responses, increased $T$ cell production, reduced inflammatory responses, and a decreased risk of illness [912]. This appears to have a plateau effect as the level of exertion, both in intensity and volume, approaches extremes. At these levels of exercise, the benefits seem to vanish. Extreme levels of exertion may even be a detrimental to the immune system. Innate immunity, including neutrophil respiratory bursts and NK-cell activity, appears to decrease in the setting of heavy exercise [13]. This seems to be the same for our adaptive immune response. PreitoHinojosa et al. found that naïve T cell numbers and thymic output were severely reduced in elite athletes. Furthermore, their immune systems mirrored those of a much older patient population. The impact of these changes on myocarditis risk remains to be elucidated [14].

The most common clinical presentation of an athlete with suspected myocarditis is a recent or previous upper respiratory tract infection [3•]. Overall, symptoms tend to be highly variable and may include a preceding flu-like illness that can manifest as fevers, chills, diarrhea, or decreased appetite. Cardiac symptoms tend to take up to days to develop and include chest pain, dyspnea, and palpitations [15]. When present, patients often describe atypical chest pain aggravated when leaning the upper body forward, suggesting the presence of a pericardial effusion [3•].Symptoms of myocarditis are usually subtle. In athletes, however, these symptoms are far more likely to be noticed due to high body awareness [16]. These symptoms include a decline in physical performance, fatigue, dizziness, or new onset atrial or ventricular arrhythmias. Some active patients may even notice increased muscle soreness that is out of proportion to the level of training they have recently completed. An increase in heart rate of 5-10 beats per minute at rest may also be a subclinical sign of an ongoing inflammatory process and should be considered when evaluating an athlete $[3 \bullet]$.

\section{Diagnosis}

Multiple diagnostic modalities are available for defining and evaluating patients with suspected myocarditis, each with varying accuracies (Table 1). Myocarditis can be especially difficult to diagnose in athletic populations, as many individuals will present with rather non-specific and vague symptoms $[3 \bullet]$. Therefore, establishing a stepwise approach for proper diagnosis and risk stratification, with an emphasis on contemporary cardiac magnetic resonance (CMR) imaging techniques, is critical and may allow for better patient outcomes. When myocarditis is suspected in an athlete with cardiac symptoms, elevations in serum inflammatory markers (erythrocyte sedimentation rate, Creactive protein) and cardiac enzymes (troponin, creatine-kinase, natriuretic peptides) can help establish a diagnosis $[6 \bullet \bullet]$. Abnormalities in the patient's 
Table 1. Accuracy of various modalities in diagnosing myocarditis [38]

\begin{tabular}{lll}
\hline Diagnostic modality & Sensitivity range & Specificity range \\
Electrocardiogram changes & 47 & -- \\
Troponin & $34-53$ & $89-94$ \\
Creatine kinase MB isoform & 6 & -- \\
Antibodies to virus or myosin & $25-32$ & 40 \\
Echocardiography (ventricular dysfunction) & 69 & -- \\
Cardiac magnetic resonance & 81 & 71 \\
Myocardial biopsy (Dallas criteria) & $35-50$ & $78-89$ \\
(-) indeterminate or poor & &
\end{tabular}

electrocardiogram (ST deviation, PR depression, arrhythmias) or echocardiogram (depressed left or right ventricular ejection fraction, regional wall motion abnormalities, pericardial effusion) can further support a diagnosis of myocarditis $[6 \bullet \bullet]$. However, coronary artery disease should always be considered and excluded as a cause for the abnormal cardiac testing.

CMR has emerged as a vital tool in establishing a diagnosis in those with a clinical presentation suggestive of myocarditis as well as assessing for prognosis $[6 \bullet \bullet]$. CMR is able to evaluate wall motion and left ventricular ejection fraction, and can visualize myocardial tissue. With respect to myocarditis, CMR carries a sensitivity of $81 \%$, a specificity of $71 \%$, and a diagnostic accuracy of $79 \%$ (Table 1) [17]. Since 2009, the Lake Louise criteria has been the recommended imaging criteria for patients with suspected myocarditis [18]. It uses CMR markers that include $\mathrm{T} 2$-weighted $(\mathrm{T} 2 \mathrm{w})$ ratios, early gadolinium enhancement, and late gadolinium enhancement (LGE). T2w sequences are edema-sensitive and can depict signal increases within inflamed myocardium; however, the extent of this edema is difficult to quantify [19]. This is usually seen as a hyper intensity signal within the epicardial layer of the myocardium [6]. Early gadolinium enhancement represents increased hyperemia and vascularity in response to myocardial injury and can be seen as a hyperlucency on CMR [18]. Late gadolinium enhancement is useful for detecting myocardial scarring and fibrosis due to volume distribution of extracellular gadolinium [20]. LGE seen in myocarditis is usually visualized in a non-ischemic distribution and sometimes does not correlate with biopsy-proven myocarditis [21]. This tends to support the argument that not every case of myocarditis leads to downstream fibrosis and scarring of the myocardium.

In athletes, the presence of LGE may act as a substrate for ventricular arrhythmias such as ventricular tachycardia. It also appears that the presence of LGE on CMR may portend a worse prognosis for athletes after myocarditis. However, when found outside the clinical context of obvious myocardial inflammation, it remains unclear whether this LGE is due to myocarditis or another etiology. In a recent study, which included athletes with structurally normal hearts by echocardiography and abnormal athletic screening (electrocardiogram or previous ventricular arrhythmia), a high rate of adverse cardiovascular events were noticed in those with areas of subepicardial LGE [22]. Researchers hypothesized that these athletes underwent a course of silent 
myocarditis and simply never developed symptoms. Fibrosis was not detected on echocardiography though was noticed on CMR, which makes this imaging

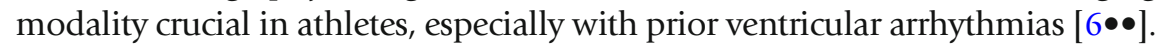
Many other studies have found a link between the presence of LGE and worse outcomes. In the ITAMY (Italian Multicenter Study on Acute Myocarditis), patients with acute myocarditis and preserved left ventricular ejection fraction showed a worse prognosis in the presence of LGE in the mid-wall layer of the anteroseptal myocardial segment [23].

Although not widely used, novel CMR techniques such as native T1 and T2 mapping have been developed to increase the sensitivity and specificity in myocarditis [24]. Both T1 and T2 mapping values are vendor and site specific. Standardization of these techniques is still needed and time will tell whether they are implemented in real-life clinical practice [6••]. It is important to note that much of the research and advancement in myocarditis is in the field of CMR. In fact, this advanced imaging modality is becoming more widely recommended as part of a more comprehensive approach to the diagnosis and management of suspected myocarditis $[6 \bullet \bullet]$.

\section{Medical therapy}

As of today, there are no randomized controlled trials for optimal medical therapy in myocarditis. The mainstay of therapy for patients with acute myocarditis should focus on guideline-directed medical treatment of heart failure and arrhythmias (Table 2) [25]. While every patient will not require a robust treatment course of medications, it is important to support a weakened left ventricle, control tachyarrhythmias, and improve a patient's volume status. In most cases myocarditis appears to resolve spontaneously with little treatment

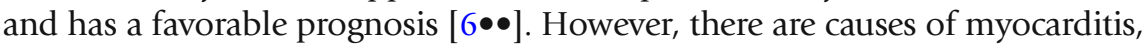
such as giant-cell myocarditis, that require disease-specific treatment to improve patient outcomes [25]. Treatment of elite athletes and recreational athletes does not vary from the general population. For the treatment of HF in the setting of

Table 2. Management guidelines for myocarditis [31, 37]

\begin{tabular}{|c|c|c|c|}
\hline Management & Recommendation & COR & LOE \\
\hline Beta blockers & Patients with reduced LVEF to prevent HF (HF stage B) & I & $\mathrm{C}$ \\
\hline ACE inhibitors & Patients with a reduced LVEF to prevent HF (HF stage $\mathrm{B} / \mathrm{C}$ ) & I & A \\
\hline ARBs & $\begin{array}{l}\text { Patients with a reduced LVEF to prevent HF who are unable to } \\
\text { take ACEI (HF stage } B / C \text { ) }\end{array}$ & I & A \\
\hline Aldosterone antagonists & Patients with NYHA class II-IV who have LVEF $\leq 35 \%$ (HF stage C) & I & A \\
\hline Sports disqualification & $\begin{array}{l}\text { Competitive athletes with probable or definite myocarditis } \\
\text { and ongoing inflammation }\end{array}$ & III & C \\
\hline Testing prior to return to exercise & $\begin{array}{l}\text { Resting echocardiogram, 24-h Holter monitor, and exercise } \\
\text { ECG no less than 3-6 months post illness }\end{array}$ & I & $\mathrm{C}$ \\
\hline $\begin{array}{l}\text { Return to exercise and competitive } \\
\text { sports }\end{array}$ & $\begin{array}{l}\text { Ventricular systolic function has returned to normal, normal } \\
\text { markers for myocardial injury, absence of arrhythmias }\end{array}$ & IIa & $\mathrm{C}$ \\
\hline
\end{tabular}


myocarditis, medications should be tailored according to the New York Heart Association (NYHA) functional class [26].

Beta-blocker treatment has numerous beneficial effects on damaged myocardium. This class of medication can improve left ventricular function, reduce future hospitalizations for heart failure, and even improve survival rates [5]. The use of beta-blockers should be avoided in those in acute decompensated heart failure and in those with early myocarditis [7]. Numerous beta-blocker options are available and the decision of which to use can be patient specific. Carvedilol has been shown to be cardio-protective in rats with autoimmune myocarditis, an effect thought to be mediated by its effect in reducing inflammation. Furthermore, it may exert anti-oxidant properties by decreasing myocardial protein carbonyl contents, a marker of cellular protein injury, in mice models. Conversely, metoprolol and propranolol were not shown to have these effects [27].

ACE Inhibitors and angiotensin-receptor blockers can slow down deleterious cardiac remodeling and progression to dilated cardiomyopathy. These medications have been shown to improve this process in animal models. The ACE inhibitor captopril significantly reduced inflammation and fibrosis in experimental autoimmune or virus-induced myocarditis [28]. Furthermore, the angiotensin-receptor blocker olmesartan improved left ventricular function and improved cardiac remodeling in mice with autoimmune myocarditis [28]. Aldosterone antagonists are recommended for systolic HF patients with NYHA functional class II to IV [7]. They are able to reduce hospital admissions for patients with HF and increase survival [26].

Nonsteroidal anti-inflammatory drugs and colchicine have very little use in patients with myocarditis. They are more commonly used as an antiinflammatory treatment in pericarditis and may be useful in those with known pericardial inflammation, chest pain, and normal left ventricular function [5].

Treatment of myocarditis with immunosuppressive agents such as azathioprine, cyclosporine, and prednisone has yielded mixed results. In patients with a virus-negative inflammatory cardiomyopathy, a combination of both corticosteroids and azathioprine was shown to improve left ventricular function over a 6-month period [29]. The use of these medications is not currently recommended, though there are specific types of myocarditis where they may be beneficial. In a multicenter study of immunosuppression utilizing cyclosporine and corticosteroids for acute GCM, patients were found to have a relatively low 1-year mortality with an acceptable safety profile [30]. Apart from heart failure therapy, managing arrhythmias and implanting intracardiac defibrillators due to myocarditis should be based on current heart failure treatment guidelines.

\section{Sports restriction and surveillance}

After a diagnosis of myocarditis is made, it is imperative for any athlete or highly active individual to refrain from physical exercise (Table 2). A proposed treatment and management algorithm for an athlete with suspected myocarditis is highlighted in Fig. 1. According to the AHA/ACC Guidelines on disqualification recommendations for competitive athletes, those with probable or definite myocarditis should not participate in sports while inflammation is present. This is independent of age, gender, and left ventricular function (Class III, Level 


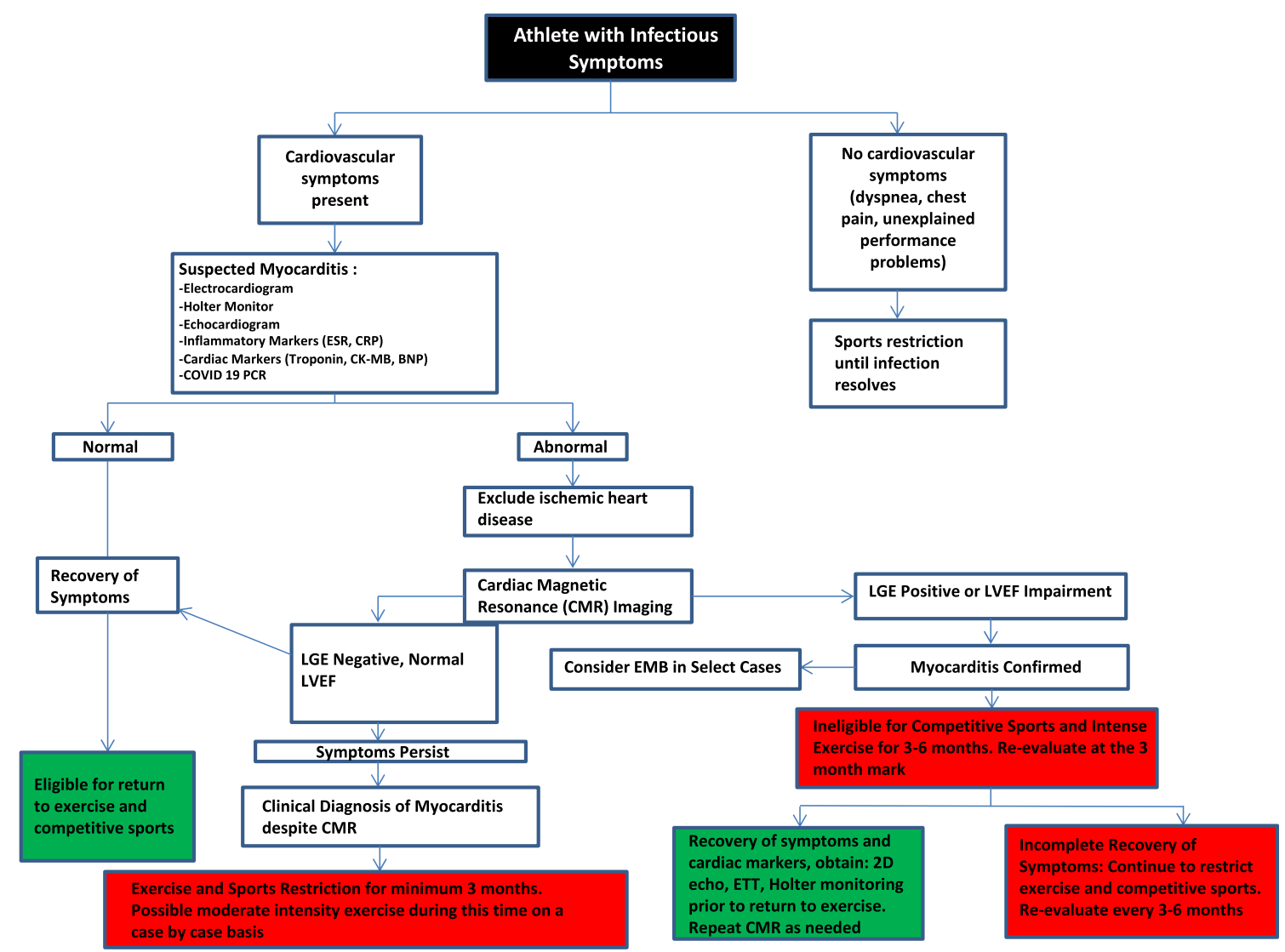

Fig. 1. Algorithm for an athlete with suspected myocarditis. ESR, erythrocyte sedimentation rate; CRP, C-reactive protein; CK-MB, creatine-kinase MB isoenzyme; BNP, brain natriuretic peptide; EMB, endomyocardial biopsy; LGE, late gadolinium enhancement; $E T T$, exercise treadmill test; LVEF, left ventricular ejection fraction.

of Evidence C). Before returning to competitive sports, the guidelines continue to recommend a resting echocardiogram, 24-h Holter monitoring, and an exercise ECG no less than 3 to 6 months after the initial illness (Class 1, Level of Evidence C) [31]. Currently, there are no risk-free levels of exercise that are recommended during this 3- to 6-month period. Certainly, high-intensity

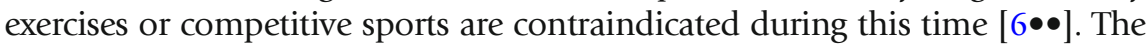
role of a less intense exercise soon after a diagnosis of myocarditis is made remains unclear. Current requirements for safe return to exercise and competition include the following: ventricular systolic function in the normal range, normalization of serum markers of myocardial injury and inflammation (troponin, brain natriuretic peptide, C-reactive protein, erythrocyte sedimentation rate), and absence of relevant arrhythmias (Class IIa, Level of Evidence C) [31].

At this time, further investigation is needed to determine whether persistent LGE in myocarditis poses a risk with ongoing sports participation and whether the risk posed is enough to merit consideration of disqualification from competitive sport $[6 \bullet \bullet]$. There is growing evidence suggesting that a decrease in LGE at follow-up may be associated with a lower event rate then an increase in burden on follow-up CMR [32]. Care providers for athletes have begun to use 
CMR both at the time of diagnosis and at follow-up to inform return to play decisions. Additionally, emphasis is placed on other means of risk stratification, as suggested by return to play guidelines, which include exercise testing that fully reproduces the demands of the athlete's sport. The ability of these mechanisms to accurately risk stratify athletes who return to sport is an area in need of further investigation.

\section{COVID-19 pandemic}

The 2020 SARS-Coronavirus-2 (COVID-19) pandemic temporarily put participation in all organized team athletics on hold. Much of the information regarding COVID-19 is changing on a daily basis and many questions remain unanswered. Most people who acquire this viral illness will remain asymptomatic or develop fairly mild symptoms [33]. However, in severe cases, mainly those requiring hospitalization, this virus can affect the heart in a similar fashion to other viral illnesses. In contrast to the typical viral pathogens that cause cardiac morbidity in $1 \%$ of hospitalized patients [8], COVID 19 appears to be significantly more cardio-toxic in this ill population [33-35]. Small studies suggest COVID-19 infection can produce cardiac manifestations, including arrhythmias and left ventricular dysfunction, in nearly $25 \%$ of hospitalized patients $[33,35]$.

The novelty of this virus and lack of robust data on its effects have reasonably resulted in return to play guidelines that are fairly conservative [36•]. As it stands, athletes that tested positive for COVID 19 and experienced symptoms at some point or another are required to abstain from sports or vigorous activity for a period of 2 weeks. After this time period and the resolution of symptoms, the current recommendation is cardiac testing, including high sensitivity troponin, an electrocardiogram, and an echocardiogram to exclude cardiac involvement, specifically myocarditis [36・]. Any abnormalities in this testing that are consistent with myocardial involvement would warrant a 3-6-month sports restriction.

The COVID 19 protocols developed by professional sports leagues as a means to resume play during this pandemic have been more involved and in some cases have required more stringent testing than has been proposed by Phelan et al. in their guideline publication. While this may be practical for professional teams, the costs associated with all this testing are likely to place a heavy burden on high school and even some NCAA sports teams. It should be expected that these guidelines will remain fluid and are likely change over time as we learn more about this virus.

\section{Conclusions}

There is not much in the way of randomized data to guide treatment specific to myocarditis in athletes. Guideline-directed therapy for heart failure is the mainstay of therapy and should be applied in cases of myocarditis with cardiac dysfunction. Physical exertion is likely a trigger for dangerous arrythmias and further propagates myocardial damage. For this reason, abstinence from sports is a critical facet of management in the initial inflammatory period. CMR has emerged as a vital tool in establishing a diagnosis in those with a clinical presentation suggestive of myocarditis as well as assessing for prognosis. More 
investigation is needed to understand the role of LGE in return to play decisions. The COVID 19 pandemic has highlighted the importance of myocarditis as a clinical diagnosis, and we await more data on the specific impact of COVID-19-associated cardiac involvement in athletes.

\section{Compliance with Ethical Standards}

Conflict of Interest

Omar Issa declares that he has no conflict of interest. Benjamin Hurwitz declares that he has no conflict of interest.

Human and Animal Rights and Informed Consent

This article does not contain any studies with human or animal subjects performed by any of the authors.

\section{References and Recommended Reading}

Papers of particular interest, published recently, have been highlighted as:

- Of importance

- Of major importance

1. Caforio ALP, Pankuweit S, Arbustini E, et al Current state of knowledge on aetiology, diagnosis, management, and therapy of myocarditis: a position statement of the European Society of Cardiology Working Group on Myocardial and Pericardial Diseases. Eur Heart $J$. 2013;34(33):2636-48, 2648a-2648d. https://doi. org/10.1093/eurheartj/eht210.

2. Martin SA, Pence BD, Woods JA. Exercise and respiratory tract viral infections. Exerc Sport Sci Rev. 2009;37(4):157-64. https://doi.org/10.1097/JES. 0b013e3181b7b57b.

3. Halle M, Binzenhöfer L, Mahrholdt H, Schindler MJ, Esefeld K, Tschöpe C. Myocarditis in athletes: a clinical perspective. Eur J Prev Cardiol. 2020:2047487320909670. https://doi.org/10.1177/ 2047487320909670 .

This is a comprehensive review of the identification, diagnosis, and treatment of myocarditis in the modern athlete. The article also highlights the guidelines for sports restriction and return-to-play as indicated by the European Society of Cardiology.

4. Mahfoud F, Gärtner B, Kindermann M, et al. Virus serology in patients with suspected myocarditis: utility or futility? Eur Heart J. 2011;32(7):897-903. https:// doi.org/10.1093/eurheartj/ehq493.

5. Kindermann I, Barth C, Mahfoud F, et al. Update on myocarditis. J Am Coll Cardiol. 2012;59(9):779-92. https://doi.org/10.1016/j.jacc.2011.09.074.

6.• Eichhorn C, Bière L, Schnell F, et al. Myocarditis in athletes is a challenge: diagnosis, risk stratification, and uncertainties. JACC Cardiovasc Imaging. 2020;13(2 Pt
1):494-507. https://doi.org/10.1016/j.jcmg.2019.01. 039.

This article focuses on how cardiac magnetic resonance plays a central role in the diagnosis and management of myocarditis. It also details how this imaging modality can add prognostic information for athletes with myocarditis.

7. Yancy CW, Jessup M, Bozkurt B, et al. 2017 ACC/AHA/ HFSA Focused Update of the 2013 ACCF/AHA Guideline for the Management of Heart Failure: A Report of the American College of Cardiology/American Heart Association Task Force on Clinical Practice Guidelines and the Heart Failure Society of Amer. Circulation. 2017;136(6):e137-61. https://doi.org/10.1161/CIR. 0000000000000509

8. Fung G, Luo H, Qiu Y, Yang D, McManus B. Myocarditis. Circ Res. 2016;118(3):496-514. https://doi.org/ 10.1161/CIRCRESAHA.115.306573.

9. Pyne DB. Regulation of neutrophil function during exercise. Sports Med. 1994;17(4):245-58. https://doi. org/10.2165/00007256-199,417,040-00005.

10. Simpson RJ, Kunz H, Agha N, Graff R. Exercise and the regulation of immune functions. Prog Mol Biol Transl Sci. 2015;135:355-80. https://doi.org/10.1016/bs. pmbts.2015.08.001.

11. Kohut ML, Arntson BA, Lee W, et al. Moderate exercise improves antibody response to influenza immunization in older adults. Vaccine. 2004;22(17-18):2298-306. https://doi.org/10. 1016/j.vaccine.2003.11.023.

12. Phillips MD, Flynn MG, McFarlin BK, Stewart LK, Timmerman KL. Resistance training at eight-repetition maximum reduces the inflammatory milieu in elderly 
women. Med Sci Sports Exerc. 2010;42(2):314-25. https://doi.org/10.1249/MSS.0b013e3181b11ab7.

13. Suzui M, Kawai T, Kimura H, et al. Natural killer cell lytic activity and CD56(dim) and CD56(bright) cell distributions during and after intensive training. J Appl Physiol. 2004;96(6):2167-73. https://doi.org/10.1152/ japplphysiol.00513.2003.

14. Prieto-Hinojosa A, Knight A, Compton C, Gleeson M, Travers PJ. Reduced thymic output in elite athletes. Brain Behav Immun. 2014;39:75-9. https://doi.org/10. 1016/j.bbi.2014.01.004.

15. JCS Joint Working Group. Guidelines for diagnosis and treatment of myocarditis (JCS 2009): digest version. Circ J. 2011;75(3):734-43. https://doi.org/10.1253/ circj.cj-88-0008.

16. Thompson PD, Dec GW. We need better data on how to manage myocarditis in athletes. Eur J Prev Cardiol. 2020:2047487320915545. https://doi.org/10.1177/ 2047487320915545

17. Lurz P, Luecke C, Eitel I, et al. Comprehensive cardiac magnetic resonance imaging in patients with suspected myocarditis: the MyoRacer-Trial. J Am Coll Cardiol. 2016;67(15):1800-11. https://doi.org/10.1016/j.jacc. 2016.02.013.

18. Pan JA, Lee YJ, Salerno M. Diagnostic performance of extracellular volume, native T1, and T2 mapping versus Lake Louise criteria by cardiac magnetic resonance for detection of acute myocarditis. Circ Cardiovasc Imaging. 2018;11(7):e007598. https://doi.org/10.1161/ CIRCIMAGING.118.007598.

19. Mayr A, Klug G, Feistritzer HJ, et al. Myocardial edema in acute myocarditis: relationship of $\mathrm{T} 2$ relaxometry and late enhancement burden by using dual-contrast turbo spin-echo MRI. Int J Card Imaging.

2017;33(11):1789-94. https://doi.org/10.1007/ s10554-017-1170-7.

20. Doltra A, Amundsen BH, Gebker R, Fleck E, Kelle S. Emerging concepts for myocardial late gadolinium enhancement MRI. Curr Cardiol Rev. 2013;9(3):18590. https://doi.org/10.2174/1573403x113099990030.

21. Friedrich MG, Sechtem U, Schulz-Menger J, et al. Cardiovascular magnetic resonance in myocarditis: a JACC White Paper. J Am Coll Cardiol. 2009;53(17):1475-87. https://doi.org/10.1016/j.jacc.2009.02.007.

22. Zorzi A, Perazzolo Marra M, Rigato I, et al.

Nonischemic left ventricular scar as a substrate of lifethreatening ventricular arrhythmias and sudden cardiac death in competitive athletes. Circ Arrhythm Electrophysiol. 2016;9(7):e004229. https://doi.org/10. 1161/CIRCEP.116.004229

23. Aquaro GD, Perfetti M, Camastra G, et al. Cardiac MR with late gadolinium enhancement in acute myocarditis with preserved systolic function. J Am Coll Cardiol. 2017;70(16):1977-87. https://doi.org/10.1016/j.jacc. 2017.08.044

24. Gati S, Sharma S, Pennell D. The role of cardiovascular magnetic resonance imaging in the assessment of highly trained athletes. JACC Cardiovasc Imaging 2018;11(2):247-59. https://doi.org/10.1016/j.jcmg. 2017.11.016.

25. Tschöpe C, Cooper LT, Torre-Amione G, Van Linthout $\mathrm{S}$. Management of myocarditis-related cardiomyopathy in adults. Circ Res. 2019;124(11):1568-83. https:// doi.org/10.1161/CIRCRESAHA.118.313578.

26. Pelliccia A, Solberg EE, Papadakis M, et al. Recommendations for participation in competitive and leisure time sport in athletes with cardiomyopathies, myocarditis, and pericarditis: position statement of the Sport Cardiology Section of the European Association of Preventive Cardiology (EAPC). Eur Heart J. 2019;40(1):19-33. https://doi.org/10.1093/eurheartj/ehy730.

27. Yuan Z, Shioji K, Kihara Y, Takenaka H, Onozawa Y, Kishimoto C. Cardioprotective effects of carvedilol on acute autoimmune myocarditis: anti-inflammatory effects associated with antioxidant property. Am J Physiol Circ Physiol. 2004;286(1):H83-90. https://doi.org/10. 1152/ajpheart.00536.2003.

28. Sukumaran V, Watanabe K, Veeraveedu PT, et al. Beneficial effects of olmesartan, an angiotensin II receptor type 1 antagonist, in rats with dilated cardiomyopathy. Exp Biol Med. 2010;235(11):1338-46. https://doi.org/ 10.1258/ebm.2010.010016.

29. Frustaci A, Russo MA, Chimenti C. Randomized study on the efficacy of immunosuppressive therapy in patients with virus-negative inflammatory cardiomyopathy: the TIMIC study. Eur Heart J. 2009;30(16):19952002. https://doi.org/10.1093/eurheartj/ehp249.

30. Cooper LT, Hare JM, Tazelaar HD, et al. Usefulness of immunosuppression for giant cell myocarditis. Am J Cardiol. 2008;102(11):1535-9. https://doi.org/10. 1016/j.amjcard.2008.07.041.

31. Maron BJ, Udelson JE, Bonow RO, et al. Eligibility and disqualification recommendations for competitive athletes with cardiovascular abnormalities: Task Force 3: Hypertrophic cardiomyopathy, arrhythmogenic right ventricular cardiomyopathy and other cardiomyopathies, and myocarditis. J Am Coll Cardiol. 2015;66(21):2362-71. https://doi.org/10.1016/j.jacc. 2015.09.035.

32. Barone-Rochette $\mathrm{G}$, Augier $\mathrm{C}$, Rodière $\mathrm{M}$, et al. Potentially simple score of late gadolinium enhancement cardiac MR in acute myocarditis outcome. J Magn Reson Imaging. 2014;40(6):1347-54. https://doi.org/10. 1002/jmri.24504.

33. Wu Z, McGoogan JM. Characteristics of and important lessons from the coronavirus disease 2019 (COVID19) outbreak in China. JAMA. 2020;323(13):1239. https://doi.org/10.1001/jama.2020.2648.

34. Clerkin KJ, Fried JA, Raikhelkar J, et al. COVID-19 and cardiovascular disease. Circulation. 2020;141(20):1648-55. https://doi.org/10.1161/ CIRCULATIONAHA.120.046941.

35. Driggin E, Madhavan MV, Bikdeli B, et al. Cardiovascular considerations for patients, health care workers, 
and health systems during the COVID-19 pandemic. $J$ Am Coll Cardiol. 2020;75(18):2352-71. https://doi. org/10.1016/j.jacc.2020.03.031.

36. Phelan D, Kim JH, Chung EH. A game plan for the resumption of sport and exercise after coronavirus disease 2019 (COVID-19) infection. JAMA Cardiol. 2020. https://doi.org/10.1001/jamacardio.2020.2136.

The article offers a focused review of return-to-play guidelines for those diagnosed with COVID-19. An algorithm is proposed for those who have developed a broad spectrum of symptoms ranging from asymptomatic infection to those athletes who have been hospitalized.

\section{Publisher's Note}

Springer Nature remains neutral with regard to jurisdictional claims in published maps and institutional affiliations. 